\title{
Modeling the contribution of the obesity epidemic to the temporal decline in sperm counts
}

\author{
Alex Kasman ${ }^{1}$, Francesco Del Giudice ${ }^{1,2}$, Eugene Shkolyar ${ }^{1}$, Angelo Porreca ${ }^{3}$, Gian Maria Busetto ${ }^{2}$, \\ Ying $\mathrm{Lu}^{4}$, Michael L. Eisenberg ${ }^{1,4}$ \\ ${ }^{1}$ Department of Urology, Stanford University School of Medicine, Stanford, California; \\ 2 Department of Maternal-Infant and Urological Sciences, "Sapienza" Rome University, Policlinico Umberto I Hospital, Rome, Italy; \\ ${ }^{3}$ Department of Urology, Policlinico Abano Terme, Abano Terme (PD), Italy; \\ ${ }^{4}$ Department of Obstetrics and Gynecology, Stanford University School of Medicine, Stanford, California; \\ ${ }^{5}$ Department of Biomedical Data Science, Stanford University School of Medicine, Stanford, California.
}

\begin{abstract}
Summary $\quad$ Objective: Total sperm count (TSC) has been declining worldwide over the last several decades due to unknown etiologies. Our aim was to model the contribution that the obesity epidemic may have on declining TSC.

Materials and methods: Obesity rates were determined since 1973 using the WHO's Global Health Observatory data. A literature review was performed to determine the association between TSC and obesity. Using the measured obesity rates and published TSC since 1973, a model was created to evaluate the association between temporal trends in obesity/temperature and sperm count.

Results: Since 1973, obesity prevalence in the United States was increased from $41 \%$ to $67.9 \%$. A review of the literature showed that body mass index (BMI) categories 2, 3, and 4 were associated with TSC (millions) of 164.27, 155.71, and 142.29, respectively. The contribution to change over time for obesity from 1974 to 2011 was modeled at 1.8\%. When the model was changed to represent the most extreme possible contribution to obesity reported, the modeled change over time rose to $7.2 \%$. When stratified according to fertility status, the contribution that BMI had to falling sperm counts for all comers was $1.7 \%$, while those presenting for fertility evaluation was $2.1 \%$. Conclusions: While the decline in TSC may be partially due to rising obesity rates, these contributions are minimal which highlights the complexity of this problem.
\end{abstract}

KEY WORDS: Obesity; Sperm count; Total sperm count; Semen analysis.

Submitted 16 September 2020; Accepted 15 October 2020

\section{INTRODUCTION}

Infertility remains an important public health concern with an estimated $15 \%$ of couples unable to conceive after 1 year of trying and therefore are labeled infertile with up to $50 \%$ having a male factor etiology $(1,2)$. A such, semen analysis remains an important component of a couple's fertility evaluation (3). With this knowledge, the overall decline in sperm count worldwide is worrisome and requires further attention. A large meta-analysis of 185 studies and data from over 42,000 men, demonstrated a $50 \%$ decline in sperm concentration and counts over the past 40 years (4). Additionally, several other studies in specific populations/countries have identified similar findings (5-10). However, the underlying cause or causes of the decline remains unknown. Given the complexity of spermatogenesis, there are likely multiple mechanisms behind declining sperm counts (e.g. environmental effects of chemical exposure, endocrine disruption, etc.) (11-14). Over the past four decades, the prevalence of obesity has increased over $50 \%$ in the world. As the obesity epidemic continues to worsen, the effect it may have on fertility has been increasingly investigated and several studies have been published on the topic. A systematic review by Guo et al., showed that overall for every five unit increase in BMI there was a 2.4\% drop in sperm count (15). Additionally, a recent large observational study of 3,966 sperm donors showed a significant decrease in sperm count for overweight and obese men (16). However, the overall contribution the obesity epidemic has to falling total sperm counts remains unknown. Given the public health implications of falling sperm counts, understanding the potential contributions of varying etiologies may have remains important. In the current study, we sought to model the potential contribution the US obesity epidemic could have to sperm counts over the past four decades.

\section{Materials ANd Methods}

This systematic review was conducted according to the Systematic Review and Meta-analysis Of Observational Studies in Epidemiology (MOOSE) guidelines (17).

The research question was established based on the following PICO criteria: what is the contribution of the obesity epidemic to the temporal decline in sperm counts? Furthermore, our goal was to explore the weighted influence of the US obesity on total sperm counts over the last four decades.

Obesity rates across the world were determined for the last four decades starting in 1973 up to 2011 using the World Health Organization's (WHO) Global Health Observatory (GHO) data (https://www.who.int/gho/ncd/ risk_factors/overweight/en/). Obesity rates were quantified using body mass index (BMI). The dates, 1973-2011, were selected based

No conflict of interest declared. 
on the real world measured sperm count data from the systematic review done by Levine et al. (4).

To determine the contribution that obesity has, on average, to total sperm count (TSC) we performed a systematic review of the literature in PubMed, Embase, and Cochrane from 1973-2011, without language restriction, to identify studies that examined infertility and/or male factor infertility in relation to the risk of mortality. The reference lists of the included studies were also screened for relevant articles. Original population-based retrospective cohort studies as well as cross-sectional and case-control cohort studies were included and critically evaluated (Level of Evidence: III-2, III-3). Case reports, abstracts and meeting reports were excluded from the analysis. Search terms included but were not limited to: primary field: body mass index or BMI, obesity, overweight AND, infertility, subfertility, semen parameters, or sperm parameters, sperm count, semen quality, sperm quality; secondary fields: oligospermia, azoospermia, oligozoospermia.

A total of 26 studies were identified that examined obesity's impact on male fertility. Six of these studies were excluded as they did not report total sperm count. From the remaining 20 studies, BMI was categorized according to healthy weight (BMI 18.5-24.9), overweight (BMI 2529.9), and obese (BMI > 30) using the Center for Disease Control's standard definition (https://www.cdc.gov/obesity/adult/defining.html). After categorization, a further 7 studies were eliminated due to overlapping BMI categories (e.g. TSC reported together for categories 3 and 4). From these remaining 14 studies, data was extracted to obtain the average TSC for each BMI category across studies with larger studies having a higher weight (Table 1).

To assess the risk of bias (RoB), all included reports were independently reviewed using the "Quality Assessment Tool for Observational Cohort and CrossSectional Studies", provided by the National Institute of Health (NIH), by assessing the potential risk for selection bias, information bias, measurement bias, or confounding bias (confounding bias includes cointerventions, differences at baseline in patient characteristics, and other issues as shown in Supplementary Table 1) (18). Studies were rated as good, fair, and poor quality, where high risk of bias translated to a rating of poor quality ("-") and low risk of bias translated to a rating of good quality ("+"). No study was considered to be seriously flawed according to the aforementioned criteria. Studies' risk of performance bias was low overall with absence of attrition bias due to incomplete outcome data across all the studies.

Annual/Decade rates of body mass index categories (i.e. normal, overweight, obese) were obtained from the WHO for 1973 and 2011. For each year, we used our calculated association
Table 1.

Studies utilized for obesity effect on sperm count.

\begin{tabular}{|l|c|l|}
\hline Category & N & Studies \\
\hline Obese & 11504 & $\begin{array}{l}\text { Belloc (2014), Paash (2010), Shayeb (2011), Aggerholm (2008), } \\
\text { Duits (2010), Xiao (2013), Macdonald (2012), Chavarro (2010), } \\
\text { Andersen (2015), Hajshafiha (2013), Vignera (2012), Gutorova (2014), } \\
\text { Ma (2019) }\end{array}$ \\
\hline Extreme obese & 297 & Hammiche (2012) \\
\hline USA & 360 & Chavarro (2010) \\
\hline Europe & 8643 & $\begin{array}{l}\text { Belloc (2014), Paasch (2010), Shayeb (2011), Aggerholm (2008), } \\
\text { Duits (2010), Anderson (2015), Vignera (2012) }\end{array}$ \\
\hline Asia & 1304 & Gutorova (2014), Ma (2019), Xiao (2013), \\
\hline New Zealand & 372 & Macdonald (2012) \\
\hline All comers & 2852 & Paasch (2010), Aggerholm (2008), Vignera (2012), Gutorova (2014) \\
\hline Fertillty evaluations & 8652 & $\begin{array}{l}\text { Belloc (2014), Shayeb (2011), Duits (2010), Xiao (2013), } \\
\text { Macdonald (2012), Chavarro (2010), Andersen (2015), Hajshafiha (2013) }\end{array}$ \\
\hline
\end{tabular}

between BMI category and sperm count to determine the average sperm count based on annual BMI. BMI category, the TSC was then multiplied by the appropriate obesity rate and a TSC for obesity was obtained for that time period (e.g. 1973 or 2011). The rates between 1973 and 2011 were then compared and a percent change over time was calculated. Over all years, we could then evaluate changes in sperm count based on temporal trends in obesity over time. TSC was then categorized according to obesity, most extreme BMI contribution (e.g. the study reporting the strongest association between BMI and TSC), region, and fertility status (unknown fertility versus those presenting for fertility evaluation). Regional areas (USA, Europe, Asia,

Supplementary Table 1.

Risk assessment of individual studies according to "Quality Assessment Tool for Observational Cohort and Cross-Sectional Studies".

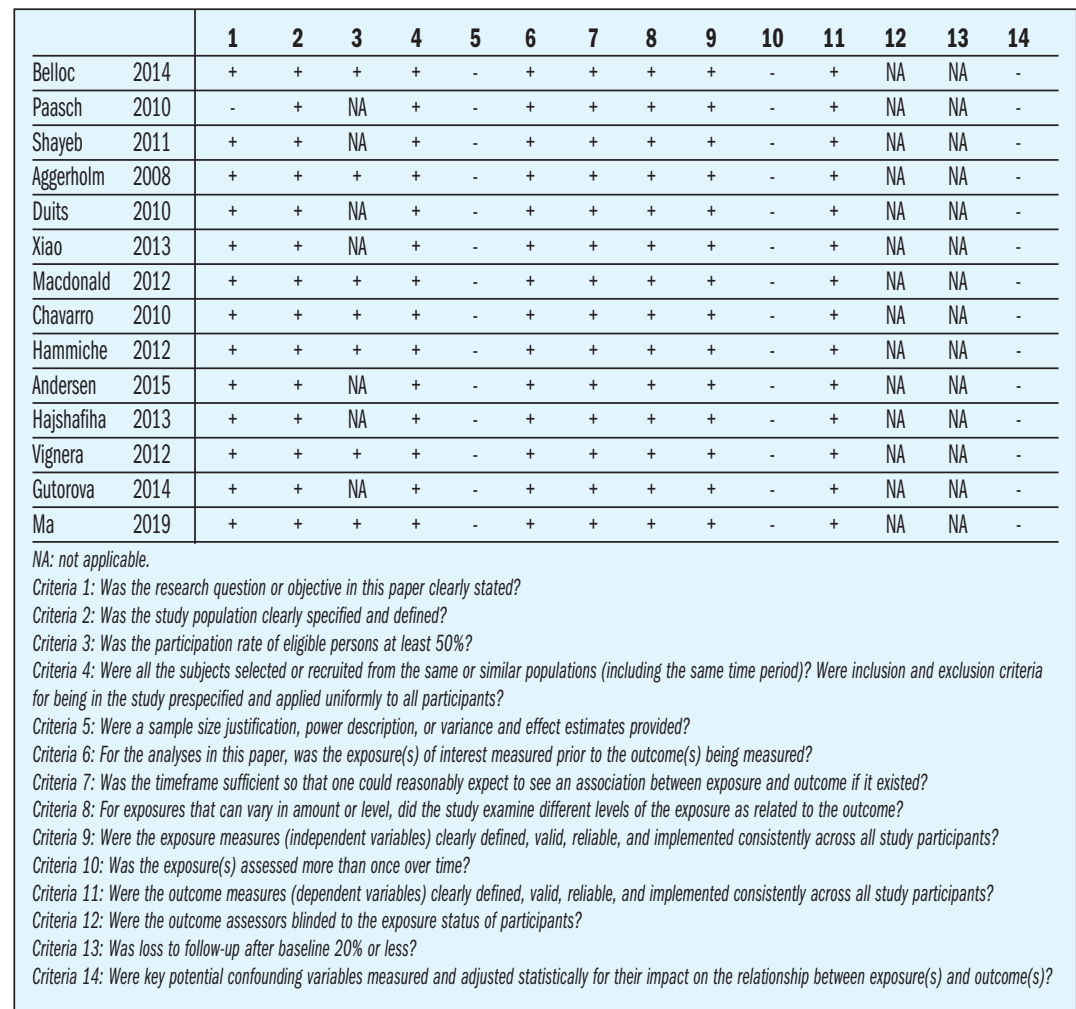


and New Zealand) were chosen based on those regions sampled in the 14 studies used. $\mathrm{P}<0.05$ were considered significant.

\section{RESULTS}

The average total sperm count (TSC, millions) for increasing BMI categories 2 (normal), 3 (overweight), 4 (obese) were 164.3, 155.7, and 142.3. The average TSC (millions) for individuals above normal BMI range (e.g. categories 3 and 4) was 149. There was not enough data present in the literature for a TSC to be calculated for BMI category 1 (underweight).

Obesity has increased in prevalence of the past 40 years. In 1973, 59\% of men were normal weight and 41\% were obese. In contrast, in 2011 (the most recent year with available data), 32.1\% were normal with $67.9 \%$ obese. Averaged across all studies, BMI categories 2, 3, and 4 were associated with TSC (millions) of 164.27, 155.71, and 142.29, respectively. The most extreme association between BMI and sperm count reported TSC (millions) of 68.6, 49.6, and 45.9 for BMI categories 2, 3, and 4, respectively (19). Overall, the contribution to change over time for obesity from 1973 to 2011 was calculated at $1.8 \%$ (Figure $2 \mathrm{a}$ ). When the model was changed to represent the most extreme possible contribution to obesity

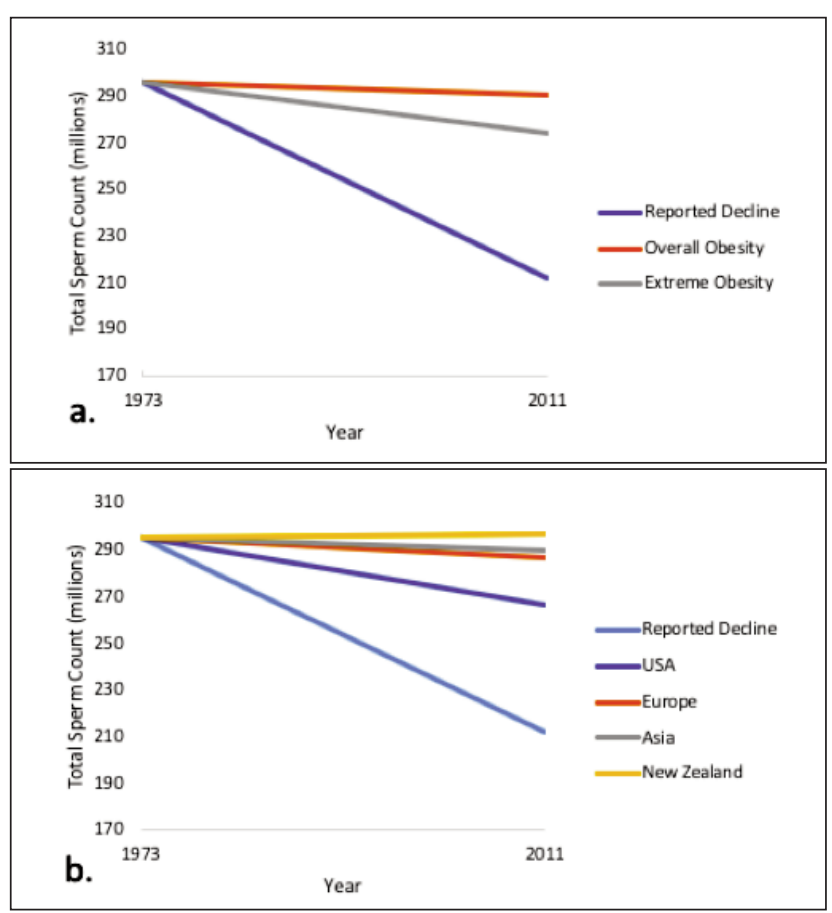

Figure 1.
PRISMA flow diagram.

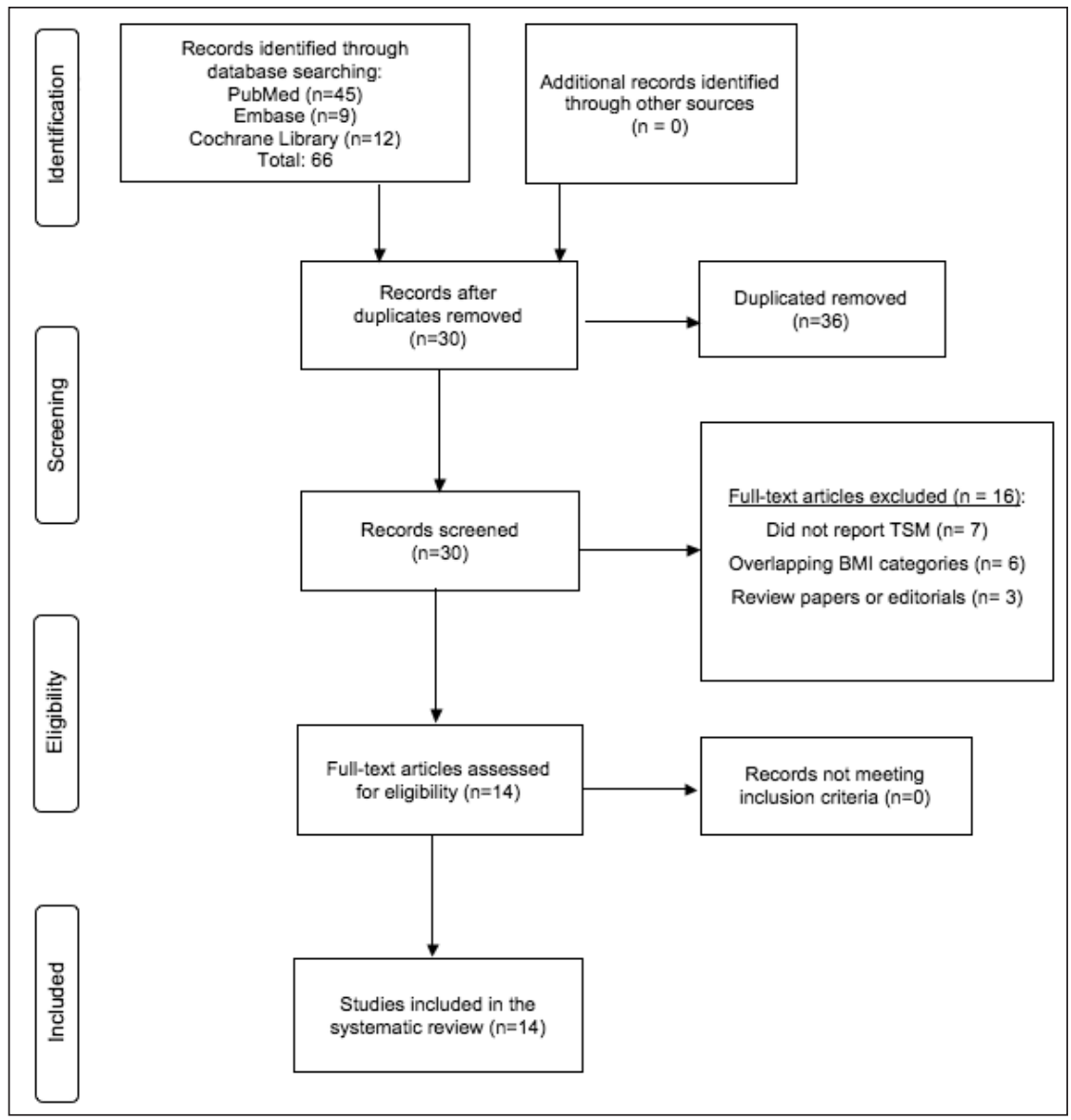

reported in any given study, the modeled change over time rose to $7.2 \%$ (Figure $2 \mathrm{a}$ ). When modeled based on regional BMI, the change for USA was 9.9\%, Europe 3.1\%, Asia $1.9 \%$, and New Zealand $-0.4 \%$ (Figure $2 b$ ). When stratified according to fertility status, the contribution that BMI had to falling sperm counts for men with unknown fertility status was 1 . $7 \%$ while those presenting for fertility evaluation was 2.1\% (Figure 2c).

\section{Figure 2.}

Model of obesity effect on sperm count stratified by overall obesity effect and largest obesity effect:

(a) fertility status known versus unknown;

(b) and region;

(c) Reported decline for all models is based on Levine et al. (Levine, Jørgensen, Martino, et al., 2017).

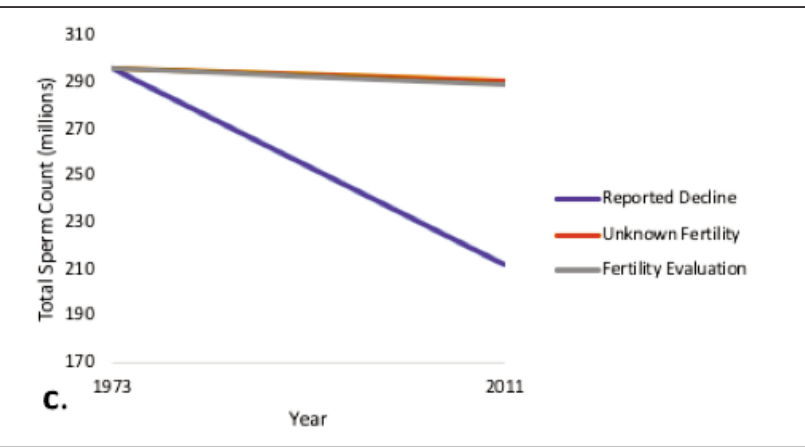




\section{Discussion}

The current report demonstrates the modest impact increasing rates of obesity may have on reported decline in semen quality. Increasing obesity rates were shown to have a small (1-10\%), though measurable contribution to the overall decline with the most measured effect, logically, observed at the extreme end of obesity's contribution. Additionally, the countries with higher obesity rates were shown to have a larger $(\sim 10 \%)$, though still modest, contribution to the reported 50\% TSC decline over the past half century. When the obesity group was stratified by fertility status, the effect did decrease in observed men with unknown fertility versus those presenting for fertility evaluation. Overall, the contributions of rising obesity rates on declining TSC appear to be individually small and suggest that the etiology for reported declines in semen quality are likely multifactorial.

As the obesity epidemic continues to worsen globally, the health effects of each continue to gain importance (2023). Additionally, during this time period, global sperm counts have been observed to be declining with unknown mechanisms (4-8). Obesity has been postulated to be one of the mechanisms driving this especially given its implications for overall health (24). Indeed, a number of primary studies have demonstrated that as an individual's BMI increases that sperm analysis parameters are affected (25-27). However, it should be noted that not all studies have found an impactful reduction in semen parameters in obese men, including a large systematic review by MacDonald, et al. $(28,29)$.

The etiology of this potential relationship is likely multifactorial which may explain the small effect that was measured in the current model. Increasing obesity has been associated with altered levels of both sex hormone binding globulin and testosterone as well as an increased estradiol to testosterone ratio (30-33).

Additionally, there is increased conversion of testosterone to estradiol in the setting of increased adiposity (34). All of these hormonal changes may ultimately lead to a negative effect downstream on spermatogenesis through the hypothalamic-pituitary-gonadal axis. While this may be a potential way in which sperm analysis parameters may be affected by obesity, the underlying mechanism through which increased adiposity could lead to impaired spermatogenesis is unknown. In addition, the additional body mass may insulate the scrotum contributing to rising scrotal temperature and lower sperm production.

The current model has several other limitations. The model itself is based on data from literature review and therefore is prone to both the bias of suitable articles for data extraction as well as the bias of the primary study itself. Additionally, a number of assumptions for the obesity model were made including that the measured effect of obesity overtime is constant. While other factors have been postulated to lead to declining sperm counts (e.g. chemical exposures), rigorous longitudinal surveillance did not allow modeling.

\section{Conclusions}

The current report demonstrates the modest contribution that obesity may have on declining total sperm counts and highlights the complex nature of infertility. Further studies are needed to examine the underlying mechanisms behind declining total sperm counts as this has large public health implications.

\section{References}

1. Thoma M, McLain A, Louis JF, et al. The prevalence of infertility in the United States as estimated by the current duration approach and a traditional constructed approach. Fertil Steril. 2014; 99:1324-1331.

2. Louis J, Thoma M, Sorensen D, et al. The prevalence of couple infertility in the United States from a male perspective : evidence from a nationally representative sample. Andrology. 2013; 1:741-748.

3. Oehninger $S$, Ombelet $W$. Limits of current male fertility testing. Fertil Steril. 2019; 111:835-841.

4. Levine H, Jørgensen N, Martino-Andrade A, et al. Temporal trends in sperm count: A systematic review and meta-regression analysis. Hum Reprod Update. 2017; 23:646-659.

5. Huang C, Li B, Xu K, et al. Decline in semen quality among 30,636 young Chinese men from 2001 to 2015. Fertil Steril. 2017; 107:83-88.e2.

6. Centola GM, Blanchard A, Demick J, et al. Decline in sperm count and motility in young adult men from 2003 to 2013: Observations from a U.S. sperm bank. Andrology. 2016; 4:270-276.

7. Sengupta P, Nwagha U, Dutta $S$, et al. Evidence for decreasing sperm count in african population from 1965 to 2015. Afr Health Sci. 2017; 17:418-427

8. Mishra P, Negi MPS, Srivastava M, et al. Decline in seminal quality in Indian men over the last 37 years. Reprod Biol Endocrinol. 2018; 16:1-9.

9. Craig JR, Jenkins TG, Carrell DT, Hotaling JM. Obesity, male infertility, and the sperm epigenome. Fertil Steril. 2017; 107:848-859.

10. Kasman AM, Del Giudice F, Eisenberg ML. New insights to guide patient care: the bidirectional relationship between male infertility and male health. Fertil Steril. 2020; 113:469-477.

11. Eisenberg ML, Li S, Cullen MR, et al. Increased risk of incident chronic medical conditions in infertile men : analysis of United States claims data. Fertil Steril. 2015; 105:629-636.

12. Mehrpour O, Karrari P, Zamani N, et al. Occupational exposure to pesticides and consequences on male semen and fertility: A review. Toxicol Lett. 2014; 230:146-156.

13. Skakkebaek NE, Rajpert-De Meyts E, Buck Louis GM, et al. Male reproductive disorders and fertility trends: influences of environment and genetic susceptibility. Physiol Rev. 2016; 96:55-97.

14. Busetto G, Del Giudice F, Virmani A, et al. Body mass index and age correlate with antioxidant supplementation effects on sperm quality: Post hoc analyses from a double-blind placebo-controlled trial. Andrologia. 2020; 52:e13523.

15. Guo D, Wu W, Tang Q, et al. The impact of BMI on sperm parameters and the metabolite changes of seminal plasma concomitantly. Oncotarget. 2017; 8:48619-48634.

16. Ma J, Wu L, Zhou Y, et al. Association between BMI and semen quality: An observational study of 3966 sperm donors. Hum Reprod. 2019; 34:155-162.

17. Group M of OS in E (MOOSE). Meta-analysis of observational studies in epidemiology: a proposal for reporting. JAMA. 2000; 283:2008-2012. 
18. National Institute of Health and Department of Health and Human Services, Quality assessment tool for observational cohort and cross-sectional studies.

19. Hammiche F, Laven JSE, Twigt JM, et al. Body mass index and central adiposity are associated with sperm quality in men of subfertile couples. Hum Reprod. 2012; 27:2365-2372.

20. Afshin A, Forouzanfar MH, Reitsma MB, et al. Health effects of overweight and obesity in 195 countries over 25 years. N Engl J Med. 2017; 377:13-27.

21. Parmesan C, Yohe G. A globally coherent fingerprint of climate change. Nature. 2003; 421:37-42.

22. Del Giudice F, Kasman A, Ferro M, et al. Clinical correlation among male infertility and overall male health: A systematic review of the literature. Investig Clin Urol. 2020; 61:355-371.

23. Del Giudice F, Kasman A, De Barardinis E, et al. Association between male infertility and male-specific malignancies: systematic review and meta-analysis of population-based retrospective cohort studies. Fertil Steril. 2020; ePub ahead.

24. Heymsfield SB, Wadden TA. Mechanisms, Pathophysiology, and Management of Obesity. N Engl J Med. 2017; 376:254-266.

25. Jensen TK, Andersson AM, Jørgensen N, et al. Body mass index in relation to semen quality and reproductive hormones among 1,558 Danish men. Fertil Steril. 2004; 82:863-870.

26. Belloc S, Cohen-Bacrie M, Amar E, et al. High body mass index has a deleterious effect on semen parameters except morphology: Results from a large cohort study. Fertil Steril. 2014; 102:1268-1273.

27. Eisenberg ML, Kim S, Chen Z, et al. The relationship between male BMI and waist circumference on semen quality: Data from the LIFE study. Hum Reprod. 2014; 29:193-200.
28. Duits FH, Van Wely M, Van Der Veen F, Gianotten J. Healthy overweight male partners of subfertile couples should not worry about their semen quality. Fertil Steril. 2010; 94:1356-1359.

29. MacDonald AA, Herbison GP, Showell M, Farquhar CM. The impact of body mass index on semen parameters and reproductive hormones in human males: A systematic review with meta-analysis. Hum Reprod Update. 2009; 16:293-311.

30. Macdonald AA, Stewart AW, Farquhar CM. Body mass index in relation to semen quality and reproductive hormones in New Zealand men: a cross-sectional study in fertility clinics. Hum Reprod. 2013; 28:3178-3187.

31. Chavarro JE, Toth TL, Wright DL, et al. Body mass index in relation to semen quality, sperm DNA integrity, and serum reproductive hormone levels among men attending an infertility clinic. Fertil Steril. 2010; 93:2222-2231.

32. Ehala-Aleksejev K, Punab M. The different surrogate measures of adiposity in relation to semen quality and serum reproductive hormone levels among Estonian fertile men. Andrology. 2015; 3:225-234.

33. Keskin M, Budak S, Aksoy E, et al. Investigation of the effect of body mass index (BMI) on semen parameters and male reproductive system hormones. Arch Ital Urol Androl. 2017; 89:219-221.

34. Michalakis K, Mintziori G, Kaprara A, et al. The complex interaction between obesity, metabolic syndrome and reproductive axis: A narrative review. Metabolism. 2013; 62:457-478.

35. Levine H, Jørgensen N, Martino A, et al. Temporal trends in sperm count: a systematic review and meta-regression analysis. Hum Reprod Update. 2017; 23:646-659.

\section{Correspondence}

Alex Kasman, MD, MS

Eugene Shkolyar, MD

Angelo Porreca MD

Department of Urology, Policlinico Abano Terme, Abano Terme (PD) (Italy)

Francesco Del Giudice, MD

Gian Maria Busetto MD, PhD

Department of Maternal-Infant and Urological Sciences, "Sapienza" Rome University, Policlinico Umberto I Hospital, Rome (Italy)

Ying Lu, PhD

Department of Biomedical Data Science, Stanford University School of Medicine, Stanford (California)

Michael L. Eisenberg, MD (Corresponding Author)

eisenberg@stanford.edu

Department of Urology, Stanford University School of Medicine,

300 Pasteur Dr., S285, Stanford, California 94305-5118 